\title{
Mitochondrial Fatty Acid $\beta$-Oxidation in the Human Eye and Brain: Implications for the Retinopathy of Long-Chain 3-Hydroxyacyl-CoA Dehydrogenase Deficiency
}

\author{
TIINA TYNI, ANDERS PAETAU, ARNOLD W. STRAUSS, BRUCE MIDDLETON, AND \\ TERO KIVELÄ \\ Department of Pediatric Neurology [T.T.], Hospital for Children and Adolescents, Department of \\ Pathology [A.P.], and Department of Ophthalmology [T.K.], Helsinki University Central Hospital, 00029 \\ HUS, Helsinki, Finland; Vanderbilt Children's Hospital [A.W.S.], Nashville, TN 37232; and Centre for \\ Biochemistry and Cell Biology [B.M.], School of Biomedical Sciences, University of Nottingham Medical \\ School, Nottingham, NG7 2UH, UK
}

\begin{abstract}
The retinal pigment epithelium (RPE) and the choriocapillaris are affected early in the retinopathy associated with long-chain 3-hydroxyacyl-CoA dehydrogenase (LCHAD) deficiency. RPE in culture possesses the machinery needed for mitochondrial fatty acid $\beta$-oxidation in vitro. To further elucidate pathogenesis of LCHAD retinopathy, we performed immunohistochemistry of the human eye and brain with antibodies to $\beta$-oxidation enzymes. Human eye and brain sections were stained with antibodies to medium-chain (MCAD) and very long-chain acyl-CoA dehydrogenase (VLCAD), short-chain 3-hydroxyacyl-CoA dehydrogenase (SCHAD), and mitochondrial trifunctional protein (MTP) harboring LCHAD. Antibodies to 2-methyl-3-hydroxybutyrylCoA dehydrogenase (MHBD) and cytochrome $c$ oxidase subunit I (COX I) were used as a reference. VLCAD, MTP, MCAD, SCHAD, MHBD, and COX I antibodies labeled most retinal layers and tissues of the human eye actively involved in oxidative metabolism (extraocular and intraocular muscle, the RPE, the corneal endothelium, and the ciliary epithelium). MTP and COX I antibodies labeled the inner segments of photoreceptors. The choriocapillaris was labeled only with SCHAD and MCAD
\end{abstract}

ABSTRACT

antibodies. In the brain, the choroid plexus and nuclei of the brain stem were most intensely labeled with $\beta$-oxidation antibodies, whereas COX I antibodies strongly labeled neurons in several regions of the brain. Mitochondrial fatty acid $\beta$-oxidation likely plays a role in ocular energy production in vivo. The RPE rather than the choriocapillaris could be the critical affected cell layer in LCHAD retinopathy. Reduced energy generation in the choroid plexus may contribute to the cerebral edema observed in patients with $\beta$-oxidation defects. (Pediatr Res 56: 744-750, 2004)

\section{Abbreviations}

COX I, cytochrome $\mathrm{C}$ oxidase of the respiratory chain

LCHAD, long-chain 3-hydroxyacyl-CoA dehydrogenase

MCAD, medium-chain acyl-CoA dehydrogenase

MHBD, 2-methyl-3-hydroxybutyryl-CoA dehydrogenase

MTP, mitochondrial trifunctional protein

RPE, retinal pigment epithelium

SCHAD, short-chain 3-hydroxyacyl-CoA dehydrogenase

VLCAD, very long-chain acyl-CoA dehydrogenase
Mitochondrial fatty acid $\beta$-oxidation is the major energyproducing pathway in several human tissues, especially in cardiac and skeletal muscle and the kidney. The liver converts acetyl-CoA formed by $\beta$-oxidation to ketone bodies, acetoac-

Received October 4, 2003; accepted May 6, 2004.

Correspondence: Tiina Tyni, M.D., Ph.D., Department of Pediatric Neurology, Hospital for Children and Adolescents, Helsinki University Central Hospital, 00029 HUS, Helsinki, Finland; e-mail: Tiina.Tyni@hus.fi

Supported by Grants from the Arvo and Lea Ylppö Foundation, Espoo, Finland; the Eye and Tissue Bank Foundation, Helsinki, Finland; Mary and Georg C. Ehrnrooth Foundation, Helsinki, Finland; the Finnish Medical Foundation, Helsinki, Finland; and the National Institutes of Health, Bethesda, MD.

DOI: 10.1203/01.PDR.0000141967.52759.83 etate, and $\beta$-hydroxybutyrate. Although the brain and eye mostly rely on glucose oxidation (1), during prolonged fasting, the brain is capable of utilizing ketone bodies (2).

Defects of mitochondrial fatty acid $\beta$-oxidation are an important group of inherited metabolic disorders that present in infancy or childhood with life-threatening metabolic crises. In only one of these disorders, long-chain 3-hydroxyacyl-CoA dehydrogenase (LCHAD; EC 1.1.1.211) deficiency, progressive pigmentary retinopathy occurs and is a major complication potentially leading to blindness (3). The retinopathy begins in infancy with pigment dispersion and degeneration of the retinal pigment epithelium (RPE), followed by circumscribed retinal atrophy with relative sparing of the peripheral fundus (3-6). 
Additional features are posterior staphyloma in advanced cases and developmental cataract. Treatment of LCHAD deficiency with a low-fat, high-carbohydrate diet (7) seems to markedly improve the prognosis and may even slow progression of the pigmentary retinopathy.

LCHAD is one of the three enzyme activities of the mitochondrial trifunctional protein (MTP) localized to the inner mitochondrial membrane. MTP is composed of four $\alpha$ - and $\beta$-subunits; LCHAD activity resides in the $\alpha$-subunits (8). The pathogenetic mechanisms underlying the retinopathy are unknown, so specific treatment strategies have not been developed. Previous studies suggest that fatty acid $\beta$-oxidation may have a role in metabolism of the porcine RPE and that MTP is also expressed in the human retina (9). To further elucidate the role of mitochondrial fatty acid $\beta$-oxidation in the human retina and the pathogenetic mechanisms of the retinopathy in LCHAD deficiency, we studied expression of $\beta$-oxidation enzymes in ocular tissues by immunohistochemistry. We also performed comparative immunohistochemistry of the brain, another organ protected by a blood-tissue barrier.

\section{METHODS}

The primary polyclonal antibodies used for immunohistochemistry were raised in rabbits to the following human enzymes that participate in fatty acid $\beta$-oxidation: very long-chain acyl-CoA dehydrogenase (VLCAD) (10), the MTP $(9,11)$, medium-chain acyl-CoA dehydrogenase (MCAD; EC 1.3.99.3) (10), and shortchain 3-hydroxyacyl-CoA dehydrogenase (SCHAD; EC 1.1.1.35) (10). MTP antibodies labeled both the $\alpha$ - and $\beta$-subunits of MTP. For reference, we used antibodies to other mitochondrial proteins: polyclonal antibodies to human 2-methyl-3-hydroxybutyryl-CoA dehydrogenase (MHBD; EC 1.1.1.178) and mouse MAb to subunit I of the complex IV (cytochrome $c$ oxidase; COX I; EC 1.9.3.1) of the respiratory chain (Molecular Probes Europe BV, Leiden, The Netherlands). This subunit is encoded by the mitochondrial DNA. MHBD is involved in $\beta$-oxidation of branchedchain amino acids (12). The study was approved by the institutional review boards, and it adhered to the tenets of The Declaration of Helsinki. All human studies were conducted with informed consent.

Immunohistochemistry of paraffin-embedded sections of the eye and brain. Formalin-fixed, paraffin-embedded eyes from four adults (age range 58-91 y) and two infants (6 and 9 mo of age) with well-preserved posterior segments were selected for immunohistochemistry from files of the Ophthalmic Pathology Laboratory, Helsinki University Central Hospital. The adult eyes were surgically removed because of ocular injury, uveal melanoma, or orbital extension of basocellular carcinoma. The children's eyes were removed because of retinoblastoma. In addition, immunohistochemistry was performed on eyes that were obtained 24 and $72 \mathrm{~h}$ post mortem from two children (14 and 7 mo of age, respectively) with LCHAD deficiency. Ophthalmopathologic findings of one of these patients have been reported earlier (3). The other died of cardiomyopathy secondary to the homozygosity for the common mutation G1528C. Formalin-fixed, paraffin-embedded brain sections from a 29-y-old adult and a 3-mo-old child were selected from the files of the Department of Pathology, Helsinki University Central Hospital. The adult died as a result of dissection of the aorta, and the child died as a result of complex cardiac anomaly. The brain sections were obtained 18 and $20 \mathrm{~h}$ post mortem, respectively. The sections selected were from the brain stem, cerebellum, frontal cortex, thalamus, and choroid plexus.

Five-micrometer-thick sections from the eyes and the brain were cut at regular intervals and mounted on chromium gelatin-coated glass slides $(0.05 \mathrm{~g}$ of potassium chromium(III)sulphate dodecahydrate and $0.5 \mathrm{~g}$ of gelatin in $100 \mathrm{~mL}$ of distilled water) or Superfrost glass slides (Erie Scientific Co., Portsmouth, NH). Some sections were pretreated with heat in citrate buffer ( $\mathrm{pH} 6.0$ ) at $95^{\circ} \mathrm{C}$ for $10 \mathrm{~min}$. The sections were stained with VLCAD, MTP, MCAD, SCHAD, MHBD, or COX I antibodies (diluted 1:400 to 1:1000). The staining was performed using the avidin-biotinylated peroxidase complex (ABC) method (Vectastain ABC Elite Kits, rabbit IgG and mouse IgG; Vector Laboratories, Burlingame, CA) as described previously in detail (13). For enabling evaluation of positive immunoreaction in the pigmented cells, the peroxidase reaction was developed with 3,3'-diaminobenzidine tetrahydrochloride (Sigma Chemical Co., St. Louis, MO). Melanin was then bleached from ocular sections by incubating the sections for $12 \mathrm{~h}$ with $3 \%$ (vol/vol) hydrogen peroxide and 1.0\% (wt/vol) disodium hydrogen phosphate, as described earlier (14). Sections that were incubated with normal rabbit serum or without primary antibody were used as negative controls in stainings with $\beta$-oxidation antibodies. Extraocular muscles in ocular sections and liver sections from diagnostic biopsy served as positive controls.

Immunohistochemistry of frozen ocular sections. Freshfrozen ocular tissue from eyes with well-preserved posterior segments and ciliary bodies were selected for immunohistochemistry from files of the Ophthalmic Pathology Laboratory, Helsinki University Central Hospital. The posterior segment sections were from two adults (63 and $70 \mathrm{y}$ of age) and one 10-mo-old child, and ciliary body sections were from two adults (43 and $53 \mathrm{y}$ of age). The eyes were surgically removed because of malignant ocular tumors. The sections were stained with the antibodies to human VLCAD, MTP, MCAD, SCHAD, and MHBD. Skeletal muscle samples that were obtained by diagnostic open biopsy from quadriceps femoris muscle were used as a positive control. Monoclonal rabbit antibodies to cow S100 protein (DakoCytomation, Glostrup, Denmark) were used as a positive control, and staining with no primary antibody served as a negative control.

From ocular tissue, 5 - $\mu$ m-thick sections were cut at regular intervals and mounted on chromium gelatin-coated glass slides. The sections were stored at $-20^{\circ} \mathrm{C}$. Before immunolabeling, they were treated with cold methanol for $10 \mathrm{~min}$ at $-20^{\circ} \mathrm{C}$. After washing with $\mathrm{PBS}, 2 \% \mathrm{BSA}$ (in PBS) was applied to sections for $30 \mathrm{~min}$. Sections were washed in PBS, and polyclonal primary antibody diluted 1:200 to 1:750 in 2\% BSA in PBS was applied to sections overnight at $4^{\circ} \mathrm{C}$. After washing with PBS, secondary antibody (goat anti-rabbit IgG, conjugated to FITC, absorbed with human IgG; lot F9887; 
Sigma Chemical Co.) diluted to $1: 75$ in $2 \%$ BSA in PBS was applied to the sections for $30 \mathrm{~min}$ at $37^{\circ} \mathrm{C}$. Sections were washed with PBS and water, air dried, and coated with mounting fluid that contained 1,4-diazobicyclo-(2,2,2)-octane (15). Sections were examined by epi-illuminated immunofluorescence microscopy and photographed.

\section{RESULTS}

Sections that were stained with VLCAD, MTP, MCAD, SCHAD, and COX I antibodies strongly labeled most extraocular muscles. The labeling intensity varied from fiber to fiber. Immunohistochemistry of paraffin-embedded sections with VLCAD and MTP antibodies showed labeling of extraocular nerves. MCAD antibodies labeled extraocular nerves more strongly in frozen than in paraffin-embedded sections. VLCAD, MTP, MCAD, SCHAD, and COX I antibodies labeled the tunica media of the extraocular arteries. Extraocular veins were labeled only with SCHAD antibodies. Immunolabeling of the ocular tissues in two patients with LCHAD deficiency showed essentially the same labeling pattern as in controls. There were no differences in the immunolabeling among ocular sections from children and adults. Hepatocytes of the control liver sections were labeled with all antibodies studied.

Anterior segment of the eye (cornea, iris, and ciliary body). In immunohistochemistry of paraffin sections, VLCAD, MTP, MCAD, SCHAD, MHBD, and COX I antibodies labeled the endothelium of the cornea (Table 1). These antibodies also labeled the posterior epithelium and the anterior myoepithelium (dilator muscle) of the iris. MCAD and SCHAD antibodies labeled the posterior epithelium of the iris more strongly than the anterior epithelium, whereas COX I antibodies labeled more intensely the anterior epithelium of the iris. All antibodies labeled the sphincter muscle. VLCAD, MTP, MCAD, SCHAD, MHBD, and COX I antibodies labeled both the inner (nonpigmented) and the outer (pigmented) epithelium of the ciliary body. In staining with MCAD and SCHAD antibodies, the labeling was stronger in the inner secretory epithelium. Antibodies to MTP, MCAD, COX I, and particularly SCHAD showed labeling of the ciliary muscle.

Table 1. Labeling of ocular tissues with antibodies to proteins of mitochondrial $\beta$-oxidation and to subunit I of complex IV (COX I) of the respiratory chain

\begin{tabular}{|c|c|c|c|c|c|c|}
\hline Layer & VLCAD & MTP & MCAD & SCHAD & MHBD* & COX I \\
\hline Corneal endothelium & + & ++ & + & ++ & + & $++\dagger$ \\
\hline \multicolumn{7}{|l|}{ Iris } \\
\hline Anterior epithelium & + & ++ & + & + & + & ++ \\
\hline Posterior epithelium & + & ++ & ++ & ++ & + & + \\
\hline Sphincter muscle & $(+)$ & ++ & ++ & ++ & ++ & ++ \\
\hline \multicolumn{7}{|l|}{ Ciliary body } \\
\hline Nonpigmented epithelium & + & ++ & ++ & ++ & + & ++ \\
\hline Pigmented epithelium & + & ++ & $(+) \ddagger$ & $+\ddagger$ & + & ++ \\
\hline Ciliary muscle & - & + & + & ++ & $(+)$ & ++ \\
\hline \multicolumn{7}{|l|}{ Neuroretina } \\
\hline Nerve fiber layer & $+1-$ & $++\S$ & ++ & $++\mathbb{I}$ & + & +++ \\
\hline Ganglion cell layer & + & ++ & ++ & $+\mathbb{d}$ & + & +++ \\
\hline Inner plexiform layer & $+1-$ & $+1-$ & ++ & $+\mathbb{I}$ & + & +++ \\
\hline Inner nuclear layer & $+1-$ & $+\|$ & $+1-\|$ & $+/-\mathbb{I I} \|$ & + & +++ \\
\hline Outer plexiform layer & - & - & ++ & $+\mathbb{I}$ & + & - \\
\hline Outer nuclear layer & $+1-$ & $+1-\#$ & $+1-\#$ & + +-【|\# & $+\#$ & - \\
\hline Outer limiting membrane & - & $+\#$ & $+\#$ & $+\llbracket \#$ & $+\#$ & - \\
\hline Photoreceptor layer & $+1-$ & $+* *$ & - & - & - & ++ \\
\hline Retinal pigment epithelium & + & ++ & + & + & + & ++ \\
\hline \multicolumn{7}{|l|}{ Choroid } \\
\hline Choriocapillaris & - & - & + & $+1-$ & - & - \\
\hline Larger choroidal vessels & - & - & ++ & $+1-$ & - & + \\
\hline Stroma & - & - & $+1-$ & - & - & - \\
\hline \multicolumn{7}{|l|}{ Extraocular tissues } \\
\hline Rectus muscle & + & $+\dagger \dagger$ & + & ++ & $+1-$ & ++ \\
\hline Ciliary arteries & 十抹 & + & + & ++ & $+1-$ & + \\
\hline Ciliary veins & - & - & - & ++ & - & - \\
\hline Ciliary nerves & + & $(+)$ & $(+) \neq$ & $(+) \neq \neq$ & - & - \\
\hline
\end{tabular}

$+/-$, the labeling varied from sample to sample; $(+)$ weak labeling.

* More clear in frozen sections, red blood cells also labeled.

$\dagger$ Cornea epithelium was also labeled.

$\ddagger$ Predominantly basal cytoplasm.

$\S$ In frozen sections.

If Probably Müller cell processes.

|| Müller cell cytoplasm.

\# Müller cell microvilli.

** Inner segments.

$\dagger \dagger++$ in children.

$\uparrow+$ Granular labeling. 
Posterior segment of the eye (retina, RPE, and choroid). MTP, MCAD, and SCHAD antibodies labeled most strongly the nerve fiber layer (Table 1, Fig. 1). The pattern of staining in the inner and outer nuclear layers of the retina with VLCAD, MTP, MCAD, and SCHAD antibodies suggested labeling of Müller radial glial cells. MTP and COX I antibodies and some sections that were stained with VLCAD antibodies showed labeling of the inner segments of the photoreceptors. MCAD, SCHAD, MHBD, and COX I antibodies labeled the inner plexiform layer, whereas MHBD and COX I antibodies also labeled the inner nuclear layer of the retina. All antibodies labeled the RPE. The immunoreactivity was most prominent in the RPE basal cytoplasm. Labeling of the RPE was most robust and widespread in sections that were stained with MTP and COX I antibodies. The choriocapillaris was unequivocally labeled only in sections that were stained with MCAD antibodies. The tunica media of choroidal arteries was labeled with MCAD, SCHAD, and COX I antibodies.

Immunohistochemistry of the brain. Labeling of most structures of the brain was more intense and consistent in immunostaining with COX I antibodies than with any $\beta$-oxidation antibody. Most intense labeling with $\beta$-oxidation antibodies was detected in choroid plexus (Fig. 2). MCAD antibodies particularly labeled capillaries and the tunica media of arterial walls.

Immunohistochemistry of the brain sections from the control individuals with MTP, MCAD, SCHAD, MHBD, and COX I antibodies showed labeling of the cytoplasm of neurons in the inferior olives and nuclei of the lower cranial nerves, including hypoglossal, vagal, and vestibular nerves (Table 2, Fig. 2). In staining with VLCAD antibodies, labeling of these cranial nerve nuclei varied from sample to sample. MTP, MCAD, and COX I antibodies particularly labeled the Raphe nucleus. All antibodies labeled the locus ceruleus. In the cerebellum, some Purkinje cells were labeled with MTP, MCAD, SCHAD, and COX I antibodies. MTP, MCAD, SCHAD, and COX I stainings showed patchy labeling in the epithelium of the choroid plexus. COX I antibodies labeled the myelin of corticospinal tracts. All antibodies labeled the tunica media of the cerebral arteries or arterioles.

\section{DISCUSSION}

We have shown previously that cultured porcine RPE cells possess the machinery needed in mitochondrial fatty acid $\beta$-oxidation in vitro (9). Labeling with MTP antibodies in several retinal layers was also detected immunohistochemically (9). On the basis of those findings, it was not clear, however, whether other $\beta$-oxidation enzymes are expressed in the human retina and whether substrates for $\beta$-oxidation have access to tissues protected by a blood-tissue barrier. The present study demonstrates selective labeling of neurons in the brain and of several cell types of the human eye with $\beta$-oxidation antibodies showing that these enzymes are not ubiquitously expressed in tissues protected by a blood-tissue barrier. Therefore, it is probable that mitochondrial fatty acid $\beta$-oxidation has a specific role in metabolism of several tissues of the eye.
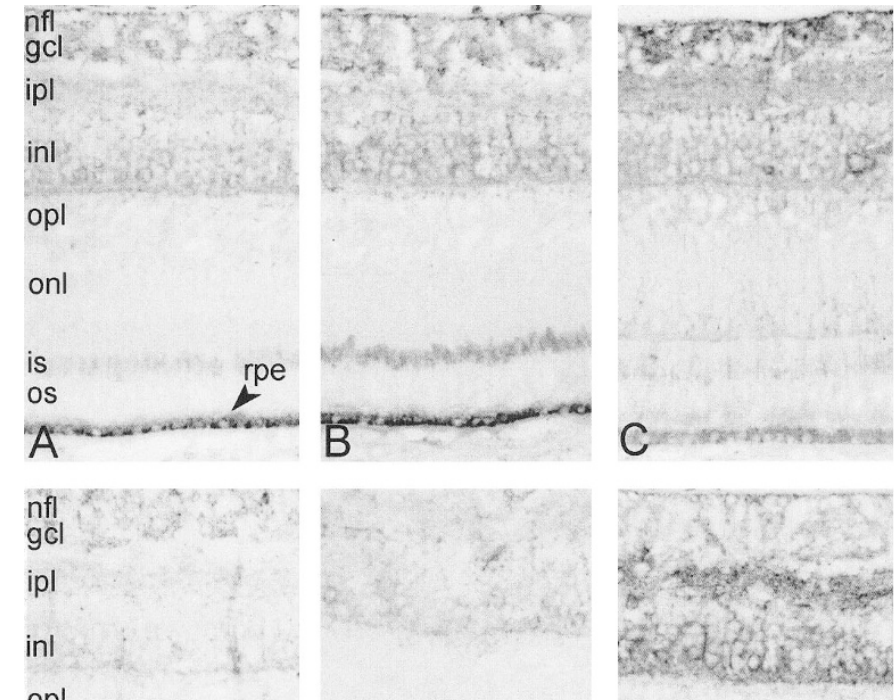

opl

onl
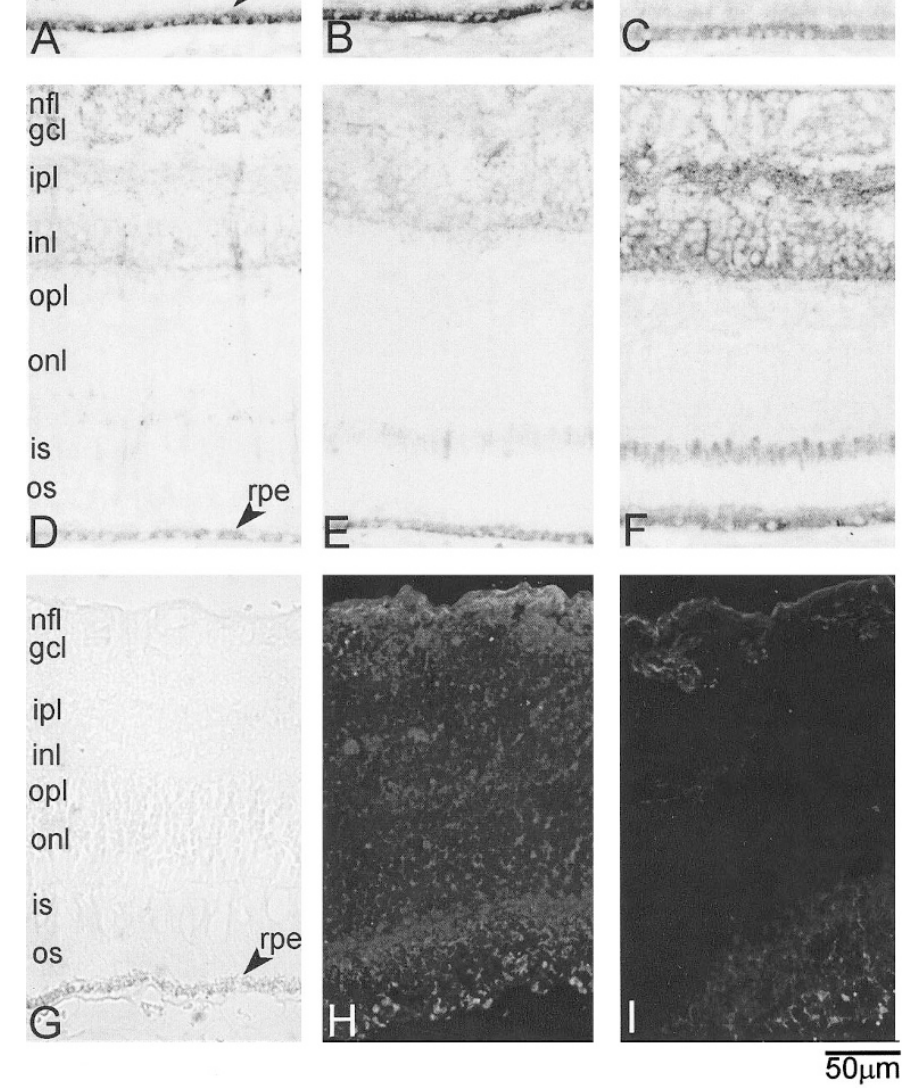

Figure 1. Immunohistochemistry of the human retinal specimen stained with mitochondrial fatty acid $\beta$-oxidation $(A-D$ and $H)$ and control antibodies $(E-G$ and $I)$ without counterstain. Sections $A-G$ are from one and sections $H$ and $I$ from another adult eye specimen. (A) VLCAD antibodies label strongly the RPE. Labeling of the nerve fiber layer ( $\mathrm{nfl}$ ), ganglion cell layer ( $\mathrm{gcl}$ ), inner nuclear layer (inl), and photoreceptor cell layer is less intense; is, inner segments of the photoreceptors; os, outer segments of the photoreceptors. (B) MTP antibodies label the RPE, photoreceptor cell layer, inner nuclear layer, ganglion cell layer, and nerve fiber layer. Labeling of the photoreceptor cells is more intense than with other $\beta$-oxidation antibodies. $(C)$ In addition to labeling of the RPE, nerve fiber layer, ganglion cell layer, and inner nuclear layer, MCAD antibodies react with the outer nuclear (onl) and outer plexiform layers (opl), consistent with labeling of the Müller cell microvilli. (D) Staining with SCHAD antibodies shows only weak labeling of the retina in paraffin-embedded sections. (E) MHBD antibodies label the RPE, inner nuclear layer, ganglion cell layer, and nerve fiber layer. $(F)$ COX I antibodies label the inner plexiform and nuclear layers more strongly than $\beta$-oxidation antibodies. $(G)$ Control section with no primary antibody. $(H)$ Staining of frozen section with SCHAD antibodies shows labeling of the nerve fiber layer, ganglion cell layer, and inner plexiform layer. Labeling of the inner nuclear layer, outer plexiform and nuclear layer, and outer nuclear layer suggests labeling of the Müller cells. (I) Control frozen section with no primary antibody shows only nonspecific labeling in photoreceptors and the inner limiting membrane. ipl, inner plexiform layer. 

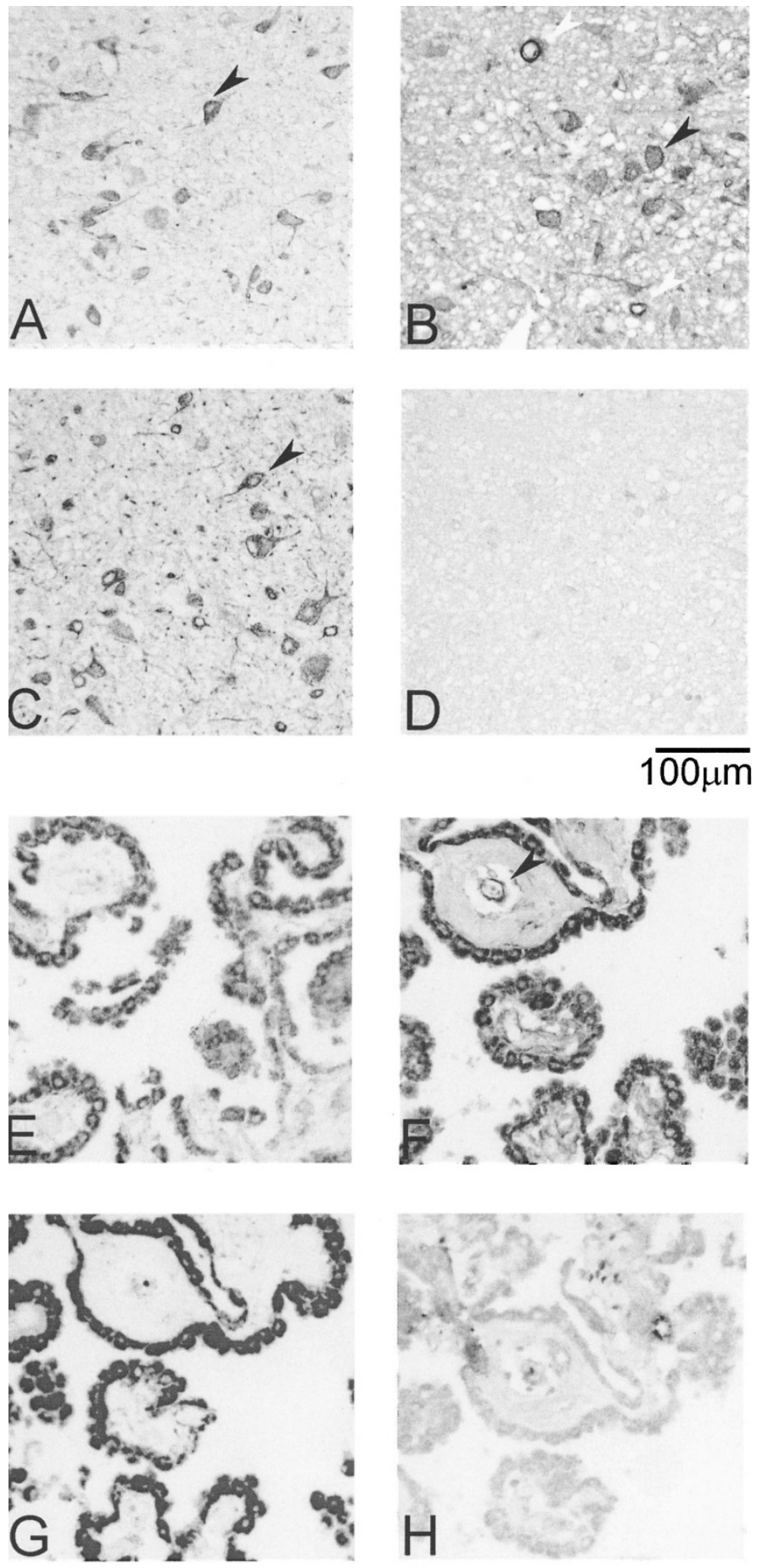

$\overline{50 \mu \mathrm{m}}$

Figure 2. Immunohistochemistry of the human hypoglossal nucleus $(A-D)$ and choroid plexis $(E-H)$ with mitochondrial fatty acid $\beta$-oxidation $(E$ and $F)$ and control antibodies $(C, D, G$, and $H$ ) without counterstain. MTP ( $A$ and $E)$, MCAD $(B$ and $F)$, and COX I $(C$ and $G)$ antibodies label the cuboidal epithelium of the choroid plexus and neurons of the hypoglossal nucleus (black arrowheads). MCAD antibodies also label the capillary wall within a choroidal villus (black arrowhead) and in the hypoglossal nucleus (white arrowhead). Control sections ( $D$ and $H$ ) stained with no primary antibody do not show any particulate labeling.

Both clinical and histopathologic evidence has shown that the layer primarily altered in the retinopathy of LCHAD deficiency is either the choriocapillaris or the RPE (4). That most $\beta$-oxidation antibodies intensely labeled the RPE and only MCAD and SCHAD antibodies labeled the choriocapillaris further suggests that the primarily affected layer in the retinopathy of LCHAD deficiency is the RPE. Although the labeling patterns of $\beta$-oxidation enzymes are not uniform, there is no conclusive difference between staining with MTP and other $\beta$-oxidation enzymes that could explain why retinopathy is a feature of only MTP deficiency. The immunohistochemistry of the neural retina shows, however, that the inner segments of photoreceptors rich in mitochondria are particularly labeled with MTP antibodies. It is possible that photoreceptor cell damage could have a contributory role in the pathogenesis of LCHAD retinopathy.

The most evident difference between staining with antibodies to $\beta$-oxidation proteins and COX I was in the sensory retina. COX I antibodies labeled the inner plexiform layer composed of the nuclei of amacrine, bipolar, horizontal, and Müller radial glial cells, whereas the labeling pattern with antibodies to fatty acid $\beta$-oxidation enzymes in this layer was compatible with labeling of only Müller cells. Because recent studies have shown that oxygen consumption in the retina is greatest in the photoreceptor layer and the inner plexiform layer (16), our findings suggest a hypothesis that LCHAD retinopathy may not result solely from depletion of the oxidative phosphorylation. This hypothesis is further supported by the fact that retinopathy is not a feature of VLCAD deficiency. Therefore, a possible pathogenetic mechanism for the retinopathy in LCHAD deficiency is either the secondary respiratory chain dysfunction detected in LCHAD deficiency (17) or potential toxicity of hydroxyacylcarnitines that accumulate in this disorder $(18,19)$. The most prominent difference between staining with antibodies to proteins involved in fatty acid and amino acid $\beta$-oxidation (MHBD) was in the extraocular muscle and nerves. MHBD antibodies did not label extraocular nerves, and labeling of extraocular muscle was sparse and inconsistent. This shows that expression of individual mitochondrial enzymes is cell specific.

Distribution of the neuronal labeling in the brain immunohistochemistry with $\beta$-oxidation antibodies relates to distribution of brain damage in Leigh syndrome, a neuropathologically or neuroradiologically detectable progressive disorder caused by several defects of oxidative phosphorylation, including respiratory chain defects and disorders of pyruvate metabolism. This suggests that these phylogenetically old regions of the brain are metabolically active. Because progressive brain damage is not a feature of $\beta$-oxidation defects, mitochondrial fatty acid $\beta$-oxidation must play a minor role in metabolism of the CNS. However, the pattern of labeling with $\beta$-oxidation antibodies is consistent with the localization of vacuolar changes in the deep gray matter, the cerebellum, and brain stem nuclei detected neuropathologically in infants with LCHAD deficiency (20).

It is interesting that the RPE, ciliary epithelium, and their equivalent in the brain, the choroid plexus, were strongly labeled with antibodies to $\beta$-oxidation enzymes. Expression of $\beta$-oxidation enzymes and COX I in the choroid plexus is not surprising because it is a metabolically highly active component of the blood-brain barrier with abundant ion channels. Of 
Table 2. Labeling of brain sections with antibodies to proteins of mitochondrial $\beta$-oxidation and to subunit I of complex IV of the respiratory chain (COX I)

\begin{tabular}{|c|c|c|c|c|c|c|}
\hline Cerebral region & VLCAD & MTP & MCAD & SCHAD & MHBD & $\mathrm{COX} \mathrm{I}$ \\
\hline \multicolumn{7}{|l|}{ Frontal cortex } \\
\hline Pyramidal cells & - & $(+) /-$ & $-*$ & $+/(+)$ & $(+) /-$ & $+/(+) \dagger$ \\
\hline Capillaries & - & - & + & - & - & - \\
\hline Thalamus (neurons) & - & + & - & + & - & + \\
\hline Nucleus subthalamicus (neurons) & - & + & $(+)$ & + & $(+)$ & ++ \\
\hline Capillaries & - & + & ++ & - & + & - \\
\hline Nucleus dentatus (neurons) & - & + & $(+)$ & - & + & ++ \\
\hline Capillaries & - & + & + & - & - & - \\
\hline \multicolumn{7}{|l|}{ Brain stem } \\
\hline Nuclei of cranial nerves (neurons) & $+/(+) \ddagger$ & + & $+/(+)$ & + & + & ++ \\
\hline Inferior olives (neurons) & - & + & $+/(+)$ & + & + & ++ \\
\hline Pons tegmentum (neurons) & - & + & $(+)$ & + & + & + \\
\hline Muscular layer of the arteries & $(+)$ & $+/(+)$ & ++ & + & + & ++ \\
\hline
\end{tabular}

$+/-$, the labeling varied from sample to sample; $(+)$ weak labeling.

* Labeled astrocytes/oligodendroglia.

$\dagger$ Labeled also the myelin of corticospinal tract.

$\ddagger$ Labeled only the neurons of nucleus hypoglossus in one section.

these cell layers, the RPE, nonpigmented ciliary epithelium, and choroid plexus contain high concentrations of mitochondria, consistent with a central role for oxidative phosphorylation in metabolism of these cells. There is also increasing evidence that the RPE cells are rich in ion channels (21-24). Coma and brain edema have been detected in patients with $\beta$-oxidation defects, even during normoglycemia (25). It has been speculated that they could result from accumulation of potentially toxic intermediates of disordered $\beta$-oxidation. Expression of $\beta$-oxidation enzymes in the human choroid plexus detected in this study by immunohistochemistry suggests that dysfunction of the blood-brain barrier also could contribute to the coma and the brain edema in patients with $\beta$-oxidation defects. Recently, expression of both muscle and liver specific isoforms of carnitine palmitoyltransferase (CPT) 1 was detected in the choroid plexus of the mouse (26). Because CPT 1 seems to be an important regulatory enzyme of the mitochondrial fatty acid $\beta$-oxidation, expression of this enzyme in the choroid plexus further confirms that $\beta$-oxidation participates in its metabolism.

\section{CONCLUSION}

Our findings further support the hypothesis that mitochondrial fatty acid $\beta$-oxidation is involved in metabolism of the RPE, which could be the cell layer that is the most severely and primarily affected in the retinopathy associated with LCHAD deficiency. Labeling of photoreceptor inner segments, particularly with MTP antibodies, could possibly explain why retinopathy is a specific feature of LCHAD deficiency. Immunohistochemistry results of the brain suggest that blood-tissue barrier dysfunction could play a role in the pathogenesis of the brain edema in $\beta$-oxidation defects.

\section{REFERENCES}

1. Berman ER 1991 Biochemistry of the Eye. Plenum Press, New York, pp 309-406

2. Owen OE, Morgan AP, Kemp HG, Sullivan JM, Herrera MG, Cahill GF Jr 1967 Brain metabolism during fasting. J Clin Invest 46:1589-1595

3. Tyni T, Kivelä T, Lappi M, Summanen P, Nikoskelainen E, Pihko H 1998 Ophthalmologic findings in long-chain 3-hydroxyacyl-CoA dehydrogenase deficiency caused by the G1528C mutation: a new type of hereditary metabolic chorioretinopathy. Ophthalmology 105:810-824

4. Tyni T, Pihko H, Kivelä T 1998 Ophthalmic pathology in long-chain 3-hydroxyacylCoA dehydrogenase deficiency caused by the G $1528 \mathrm{C}$ mutation. Curr Eye Res 17:551-559

5. Schrijver-Wieling I, van Rens GH, Wittebol-Post D, Smeitink JA, de Jager JP, de Klerk HB, van Lith GH 1997 Retinal dystrophy in long-chain 3-hydroxy-acyl-coA dehydrogenase deficiency. Br J Ophthalmol 81:291-294

6. Lawlor DP, Kalina RE 1997 Pigmentary retinopathy in longchain 3-hydroxyacylcoenzyme A dehydrogenase deficiency. Am J Ophthalmol 123:846-848

7. Gillingham MB, Connor WE, Matern D, Rinaldo P, Burlingame T, Meeuws K, Harding CO 2003 Optimal dietary therapy of long-chain 3-hydroxyacyl-CoA dehydrogenase deficiency. Mol Genet Metab 79:114-123

8. Kamijo T, Wanders RJ, Saudubray JM, Aoyama T, Komiyama A, Hashimoto T 1994 Mitochondrial trifunctional protein deficiency. Catalytic heterogeneity of the mutant enzyme in two patients. J Clin Invest 93:1740-1747

9. Tyni T, Johnson M, Eaton S, Pourfarzam M, Andrews R, Turnbull DM 2002 Mitochondrial fatty acid $\beta$-oxidation in the retinal pigment epithelium. Pediatr Res 52:595-600

10. Shekhawat P, Bennett MJ, Sadovsky Y, Nelson DM, Rakheja D, Strauss AW 2003 Human placenta metabolizes fatty acids: implications for fetal fatty acid oxidation disorders and maternal liver diseases. Am J Physiol 284:E1098-E1105

11. Carpenter K, Pollitt RJ, Middleton B 1992 Human liver long-chain 3-hydroxyacylcoenzyme A dehydrogenase is a multifunctional membrane-bound beta-oxidation enzyme of mitochondria. Biochem Biophys Res Commun 183:443-448

12. Ofman R, Ruiter JP, Feenstra M, Duran M, Poll-The BT, Zschocke J, Ensenauer R, Lehnert W, Sass JO, Sperl W, Wanders RJ 2003 2-methyl-3-hydroxybutyryl-CoA dehydrogenase deficiency is caused by mutations in the HADH 2 gene. Am J Hum Genet 72:1300-1307

13. Fuchs U, Kivelä T, Summanen P, Immonen I, Tarkkanen A 1992 An immunohistochemical and prognostic analysis of cytokeratin expression in malignant uveal melanoma. Am J Pathol 141:169-181

14. Kivelä T 1995 Immunohistochemical staining followed by bleaching of melanin: a practical method for ophthalmic pathology. Br J Biomed Sci 52:325-326

15. Johnson GD, Davidson RS, McNamee KC, Russell G, Goodwin D, Holborow EJ 1982 Fading of immunofluorescence during microscopy: a study of the phenomenon and its remedy. J Immunol Methods 55:231-242

16. Cringle SJ, Yu DY, Yu PK, Su EN 2002 Intraretinal oxygen consumption in the rat in vivo. Invest Ophthalmol Vis Sci 43:1922-1927 
17. Tyni T, Majander A, Kalimo H, Rapola J, Pihko H 1996 Pathology of skeletal muscle and impaired respiratory chain function in long-chain 3-hydroxyacyl-CoA dehydrogenase deficiency with the G1528C mutation. Neuromuscul Disord 6:327-337

18. Wojtczak L, Schonfeld P 1993 Effect of fatty acids on energy coupling processes in mitochondria. Biochim Biophys Acta 1183:41-57

19. Singh AK, Yoshida Y, Garvin AJ, Singh I 1989 Effect of fatty acids and their derivatives on mitochondrial structures. J Exp Pathol 4:9-15

20. Tyni T, Rapola J, Paetau A, Palotie A, Pihko H 1997 Pathology of long-chain 3-hydroxyacyl-CoA dehydrogenase deficiency caused by the G1528C mutation. Pediatr Pathol Lab Med 17:427-447

21. Weng TX, Godley BF, Jin GF, Mangini NJ, Kennedy BG, Yu AS, Wills NK 2002 Oxidant and antioxidant modulation of chloride channels expressed in human retinal pigment epithelium. Am J Physiol 283:C839-C849
22. Sheu SJ, Wu SN 2003 Mechanism of inhibitory actions of oxidizing agents on calcium-activated potassium current in cultured pigment epithelial cells of the human retina. Invest Ophthalmol Vis Sci 44:1237-1244

23. Rosenthal R, Strauss O $2002 \mathrm{Ca} 2+$-channels in the RPE. Adv Exp Med Bio 514:225-235

24. Hamann S, Kiilgaard JF, la Cour M, Prause JU, Zeuthen T 2003 Cotransport of $\mathrm{H}^{+}$, lactate, and $\mathrm{H}_{2} \mathrm{O}$ in porcine retinal pigment epithelial cells. Exp Eye Res 76:493-504

25. Roe CR, Coates PM 1989 Acyl-CoA dehydrogenase deficiencies. In: Scriver C, Beaudet A, Sly W, Valle D (eds) The Metabolic Basis of Inherited Diseases. McGraw-Hill, New York, pp 889-914

26. Sorensen A, Travers MT, Vernon RG, Price NT, Barber MC 2002 Localization of messenger RNAs encoding enzymes associated with malonyl-CoA metabolism in mouse brain. Brain Res Gene Expr Patterns 1:167-173 